\title{
Ressources cognitives et développement territorial : une analyse textuelle appliquée aux politiques locales de développement durable
}

\author{
Cognitive resources and territorial development: \\ textual analysis applied to local policies of sustainable \\ development
}

Gaël PlumecocQ

INRA (UMR AGIR) et LEREPS

Chargé de recherche

Chemin de Borde-Rouge

31326 CASTANET-TOLOSAN

Gael.Plumecocq@toulouse.inra.fr 
Mots clef : Analyse textuelle, Développement durable, Patrimoine, Proximité cognitive, Territoire

Keywords: Cognitive proximity, Patrimony, Sustainable development, Territory, Textual analysis

Classification JEL : Q01, R19, B41

\section{Résumé}

Cet article étudie comment un développement régional cohérent nécessite la mobilisation de ressources cognitives partagées. Dans le cas étudié - la région Nord Pas-de-Calais, cette proximité cognitive se construit via les politiques locales, sur la base du référentiel de développement durable. Pour saisir la manière dont les collectivités de la région activent cette ressource, nous avons mobilisé les outils d'analyse textuelle sur une trentaine d'entretiens auprès des acteurs publics du développement durabe. Les résultats mis en évidence suggèrent que cette proximité cognitive repose sur deux éléments fondamentaux: d'une part la mise en valeur d'un patrimoine infrastructurel territorialisé, et d'autre part la reconstruction d'une identité territoriale. Les politiques locales prennent ainsi appui sur les valeurs qui sous-tendent ces éléments ainsi que sur des modalités rhétoriques, pour impulser en profondeur une dynamique de changement, plus malaisée à mettre en ouvre partir des outils politiques habituels.

\section{Summary}

This paper focuses on how the development of a particular region consistently requires actors to share a certain level of cognitive resources. In the case studied here - the Nord Pas-deCalais region, this cognitive proximity is built via the local policies involving sustainable development. In order to comprehend the way local communities of the region activate this resource, we used textual data treatment analyzing about thirty interviews. The results suggest that this cognitive proximity relies on two fundamentals elements: on the one hand, valuing the patrimonial infrastructures of the territory; and on the other hand, rebuilding the territorial identity of the region. Then the local policies lean both on the values that underlie these elements and on rhetorical modalities, to impulse in-depth changes that would have been more difficult to implement from the usual political levers. 


\section{-1 - \\ Introduction}

Le développement durable constitue un objet de recherche relativement difficile à saisir. L'expression même peut apparaître comme une contradiction dans les termes (RIST, 1996). Dans cet article, nous partons du postulat que l'action entreprise au nom du développement durable a un sens à la fois historique et épistémique. Dans cette optique, le développement durable n'est pas donné en soi, mais résulte d'une construction sociale des acteurs qui lui donnent son sens. Nous soutenons que cette dimension historico-épistémique peut être analysée comme une ressource que nous qualifierons de «cognitive ». L'originalité de notre démarche repose sur deux éléments complémentaires: d'une part, une conception pragmatique du développement durable comme vecteur de savoirs et surtout de valeurs qui façonnent une identité territoriale et apparaissent fondamentaux en vue de la mobilisation collective; et d'autre part, une méthode permettant de mettre en évidence ces ressources cognitives à travers la manière dont les acteurs en rendent compte, au moyen d'une analyse textuelle. En nous appuyant sur l'exemple des collectivités locales de la région Nord Pas-deCalais, acteurs particulièrement légitimes en matière de développement durable, nous montrons que les ressources cognitives d'un territoire reposent sur deux éléments complémentaires: d'une part, sur l'existence d'une identité territoriale, comprise comme le fait que les individus d'une population se réclament manifestement et évidemment d'un territoire donné ; et d'autre part, sur la valorisation d'un patrimoine historique. Ces deux éléments sont étroitement reliés par les valeurs qui les sous-tendent. Ils contribuent ensemble à légitimer l'action publique en faveur du développement durable. Du point de vue de la science régionale, cela permet d'aborder la question des leviers politiques du développement régional sous une perspective rhétorique, et non plus seulement à travers les seuls outils de politique économique que sont l'incitation et la réglementation.

Nous commencerons par préciser le cadre théorique, et en particulier la manière dont nous positionnons les notions d'identité territoriale et de patrimoine au regard du concept de ressource cognitive (section 2). Nous examinerons ensuite l'histoire industrielle de la région, de façon à éclairer la manière dont émergent les valeurs régionales qui justifient que l'on puisse considérer le développement durable comme une ressource (section 3). La section 4 présente la méthodologie ainsi que les résultats préliminaires obtenus à partir de l'analyse textuelle des entretiens menés auprès des décideurs publics de la région. Ces derniers ont ensuite été commentés dans le but de reconstituer les discours locaux de développement durable et de saisir la manière avec laquelle cette ressource se construit dans l'action (section 5). Cela nous a finalement permis d'exposer la façon dont la mobilisation du développement durable dans les politiques publiques locales reconfigure les valeurs patrimoniales et identitaires du territoire (section 6). 


\section{- 2 - \\ Ressources cognitives, identité territoriale et patrimoine}

Deux éléments sont au fondement de la notion de ressource cognitive dans un contexte territorial : (i) l'identité territoriale et (ii) le patrimoine. Dans cette section, nous discutons de l'articulation théorique de ces notions.

Selon BRUNET, et al. (1992), un territoire est un système formé de cinq sous-systèmes, dans lequel coexistent un système d'appropriation par ses habitants ; un système de gestion défini par des règles juridictionnelles et administratives d'ordre politique; un système d'appartenance réelle au territoire en relation avec les phénomènes de peuplement (notamment l'habitation); un système de ressources permettant, entre autres, de légitimer le peuplement du territoire ; et enfin un système social d'interactions entre individus, par le biais d'échanges. Ces sous-systèmes ne peuvent être pensés indépendamment les uns des autres. Les notions (i) d'identité territoriale et (ii) de patrimoine nous permettent d'aborder conjointement l'ensemble des sous-systèmes territoriaux, mais également de mieux identifier les rapports coévolutifs qu'ils entretiennent. Par exemple, (i) l'identité d'un territoire peut être définie au regard des ressources culturelles mobilisées sur le territoire (GREFFE, 2005, KAHN, 2010). De ce point de vue, l'identité territoriale se construit via les interactions interindividuelles, peut impacter les règles d'administration du territoire, et fonde l'appartenance au territoire. De même, (ii) la notion de patrimoine n'est pas réductible à celle de capital, d'actif ou même de ressource. Le patrimoine est, en effet porteur d'éléments, y compris de valeur, propres à l'identité collective de ses détenteurs (BARRERE, et al., 2005, VIVIEN, 2009). Dans cette perspective, le patrimoine territorial peut justifier le système d'appropriation du territoire, être perçu comme une ressource et nécessiter des règles appropriées de gestion.

De plus, identité territoriale et patrimoine ne sont pas déconnectés. Le sentiment d'identité territoriale nécessite la reconnaissance d'éléments partagés par les populations, ce que permet d'objectiver l'existence et la reconnaissance d'un patrimoine à préserver et à valoriser. Ces éléments de partage apparaissent dans le sens que donne l'économie de la proximité (PECQUEUR, et al., 2004, TORRE, et al., 2008) à la notion de «territoire commun », c'est-à-dire fondé « à la fois sur la référence à un lien de proximité géographique [...] et à un lien de proximité organisationnelle » (TORRE, 2000, p. 411). Ces référentiels peuvent procéder de caractéristiques physiques des territoires (PECQUEUR, et al., 2004). Pour autant, ils ne se donnent pas d'emblée comme communs. Ainsi, comme le souligne TORRE, «la proximité géographique est non seulement une question de distance ou de topologie, mais encore de perception et de ressenti des acteurs [...] au-delà de son rôle de ressources recélant des potentialités, elle se pose également et avant tout comme un déterminant d'évolution des relations humaines » (TORRE, 2008, p. 332). Il existe donc un lien dynamiquement réciproque entre proximité géographique et proximité cognitive, puisque l'arrivée de nouveaux savoirs modifie la conception du territoire, la manière dont il a été institutionnalisé et les rapports organisés entre ses acteurs. De même toute modification dans l'évolution du territoire, qu'elle soit d'origine écosystémique ou anthropique implique un changement de perception de la part des individus. BEAURAIN (2008) insiste ainsi particulièrement sur la réciprocité de ce lien lorsqu'il met en évidence les mécanismes par lesquels la construction d'un territoire (partagé) s'appuie sur des ressources cognitives. A l'inverse, la proximité géographique permet de s'affranchir des contraintes de spatialité, offrant aux individus un cadre dans lequel interagir 
(TORRE, et al., 2005). La coordination des acteurs à l'intérieur d'une structure organisée nécessite également le partage d'un minimum de référentiels communs, en particulier sur le bien commun poursuivi par l'organisation (BOLTANSKI, et al., 1991).

En l'absence de cadre cognitifs ou de référentiels partagée, l'espace géographique ou la structure organisée ne portent en elles qu'un potentiel pour la coordination (TALBOT, 2008). La proximité cognitive joue donc un rôle fondamental dans et pour les autres formes de proximité. Bien loin de tenter de remettre en question la structure conceptuelle de l'économie de la proximité (BOUBA-OlGA, et al., 2008), nous nous concentrerons sur la seule proximité cognitive, que nous définirons comme l'ensemble des savoirs détenus par les acteurs, et qui peut apparaître comme une forme de proximité institutionnelle (TALBOT, 2008). Ces savoirs sont non seulement techniques (les manières de faire, les «bonnes recettes »...), mais aussi déontologiques (les valeurs morales) et ontiques (les caractéristiques des choses et des autres acteurs). Nous soutenons qu'en matière de développement durable, ces savoirs sont essentiellement pragmatiques, dans le sens où ils procèdent de la pratique des acteurs et de leurs interactions qui ont du même coup une fonction de production épistémique (NoOTEBOOM, 2000) : en agissant, les acteurs produisent également du sens. Lorsque le sens des actions est socialement accepté par chacun, l'engagement collectif dans un changement de pratiques est possible. Si la véridicité de ces savoirs n'est pas requise (DUPUY, 1989) - il suffit que les croyances dont elles sont issues convergent, la plupart du temps, l'adoption de nouvelles pratiques n'est institutionnalisée (si ce n'est possible) que lorsque la validité des savoirs est reconnue. La croyance partagée dans la véridicité des savoirs (à un méta-niveau) peut se manifester de diverses manières. On peut ainsi supposer que la mobilisation du référentiel de développement durable par les collectivités territoriales du Nord Pas-de-Calais depuis plus de vingt ans relève d'un réel engagement collectif plutôt que d'un effet de mode. Cet engagement dans la durée permet de cristalliser la confiance collective à l'épreuve de l'histoire et peut ainsi déboucher sur la création d'institutions garantissant cette confiance.

Inversement, lorsqu'une trop grande base cognitive est partagée entre les acteurs, cela peut bloquer toute action collective et conduire à l'inanité. De même que dans certains cas le défaut d'organisation ou d'institutions peut créer la nécessité de passer par d'autres modes de coordination, plus informels (BOUBA-OlgA, et al., 2008), une certaine «distanciation » dans les savoirs peut, dans certains cas où ceux-ci s'avèrent complémentaires, favoriser la coopération (NoOTeBoom, 2000, Boschma, 2005). Entre le trop et le trop peu, un niveau minimum de proximité cognitive est requis pour mener à bien l'action collective (BOSCHMA, et al., 1999, Boschma, 2005) : d'une part, la coopération n'est possible qu'entre individus partageant un minimum de caractéristiques (c'est à ce niveau que jouent les dimensions patrimoniales et identitaires du territoire); d'autre part, la coopération n'a d'intérêt qu'entre des individus suffisamment différents pour que la complémentarité de leurs différences produise un résultat relativement supérieur à la somme de ceux qu'ils obtiendraient individuellement (BOLTANSKI, et al., 1991).

\section{- 3 - \\ Perspectives historiques : une ressource latente pour le territoire}

La construction d'une ressource territoriale - ici le développement durable, ne peut se comprendre pleinement que dans une mise en perspective historique ( $c f$. Encadré). Le développement industriel qu'a connu la région Nord Pas-de-Calais permet d'analyser la manière avec laquelle l'identité territoriale se constitue en lien avec la production de richesses 
pour le territoire et ses habitants. Ce mode de développement profondément marqué par l'histoire de l'essor industriel de la région, constitue le terreau sur lequel le développement durable devient une ressource potentielle que qualifierons de « latente ».

\section{Encadré : Jalons de l'histoire du développement industrielle de la région Nord Pas-de-Calais}

- Années 1850 : découverte de gisement de charbons dans la région lensoise. Début de la période d'expansion.

- Première Guerre Mondiale : les deux tiers des 212 puits de charbon sont détruits.

- Entre deux guerre : restructuration du secteur.

- En 1940, 18 compagnies exploitent le gisement. $60 \%$ de la production nationale provient de la région.

- Période d'après-guerre : nationalisation des compagnies minières. Les richesses alors captées dans la région (patrimoine foncier ou investissements immobiliers) sont désormais redéployées sur tout le territoire national. Période productiviste: les puits sont progressivement fermés, mais les niveaux de production restent constants.

- 1970 : l'activité d'extraction est quasiment à l'arrêt.

- 1990 : fermeture du dernier puits.

D'une part, cette histoire se transmet par des canaux domestiques propres au régime de la familiarité (BOLTANSKI, et al., 1991), qui se manifeste notamment à travers la construction par les compagnies minières de lieux de vie et d'un habitat minier (cités minières, corons...), en jouant sur le double registre de la proximité organisationnelle et de la proximité géographique propre au régime paternaliste industriel du $19^{\text {ème }}$ siècle. Cet ancrage des populations dans des espaces communautaires constitutifs d'une identité, touche non seulement les mineurs eux-mêmes, mais également leurs familles qui jouent alors un rôle de réceptacle, puis de canal de transmission de cette histoire.

D'autre part, cette histoire se développe en lien étroit avec l'attachement des habitants au territoire. Celui-ci trouve sa source dans deux idées partagées par les populations (mais potentiellement contradictoires !).

- Première idée, la ressource sur laquelle s'est développée la région est une ressource naturelle (BEAURAIN, 2008). Celle-ci est à la source des richesses qui se sont pérennisées sur le territoire (réseaux de transports, infrastructures minières, bâtiments des administrations, etc.), et qui trouve sa traduction dans la richesse de l'architecture urbaine (Lille, Arras, Valenciennes...). Ce patrimoine constitue ainsi un trait d'union entre un passé illustre et des valeurs collectives toujours partagées, en particulier celles liées au travail (région industrieuse, travailleuse, productive, courageuse, volontaire, généreuse dans l'effort...).

- Seconde idée, cette histoire aboutit à une situation désastreuse en termes socioéconomiques (la ville de Valenciennes perd presque $18 \%$ de sa population entre 1970 et 1990, la pauvreté est 16 à $26 \%$ plus élevée que dans le reste du pays (GADREY, et al., 2006)), mais également en termes écologiques (la moitié des sols pollués en France sont situés dans le Nord Pas-de-Calais, 10000 hectares du territoire sont des friches industrielles, etc. (SCARWELL, et al., 2008)). Ce bilan très négatif contribue également à façonner l'identité du territoire (ZUINDEAU, et al., 2008).

L'histoire du développement économique de la région a donné lieu à divers modèles de développement local (MAILlEFERT, 2009). Pour autant, elle explique sans doute qu'une 
conscience écologique s'y soit développée plus tôt que dans d'autres régions françaises (EMELIANOFF, 2005), créant notamment le premier Parc Naturel Régional en France en 1968. Politiquement, cela se traduit par deux évènements majeurs : en 1989, l'élection de JeanLouis Borloo à la mairie de Valenciennes (il participera l'année suivante à la fondation du mouvement «Génération Ecologie »); et l'élection de Marie-Christine Blandin qui devient la première écologiste Présidente de Région en 1992. Cette dynamique est également renforcée par la création d'institution destinées à mieux appréhender les séquelles territoriales héritées de l'ère industrielle (e.g. la création en 1990 de l'Etablissement Public Foncier du Nord Pasde-Calais).

Ainsi, dès le milieu des années 1990, les collectivités de la région, sous l'impulsion de certaines d'entre elles plus innovantes, amorcent un tournant vers le développement durable ${ }^{1}$. A cette date, celui-ci n'est qu'une ressource cognitive latente. Pour autant, l'identité territoriale ainsi que les richesses (en particulier immobilières) en voies de patrimonialisation supportent déjà les valeurs partagées qui permettront, dans les décennies suivantes, de mobiliser plus efficacement cette ressource dans l'engagement collectif vers des comportements plus soutenables (BEAURAIN, 2008).

\section{- 4 - \\ Méthodes et résultats}

La méthode mobilisée tente de saisir cette activation à travers une analyse d'entretiens menés auprès des acteurs des politiques de développement durable des collectivités territoriales de la région. Après avoir détaillé la composition de l'échantillon et décrit la manière avec laquelle les entretiens ont été menés, nous présenterons la méthode d'analyse textuelle utilisée (Alceste, version 4.7 de 2002).

\subsection{Description des données}

De par son histoire, la région Nord Pas-de-Calais constitue un cas d'étude intéressant. Très tôt engagée pour le développement durable (une grande proportion des collectivité qui répondent au premier appel à projet "Agendas 21 locaux" du Ministère de l'Environnement, sont localisées dans le Nord Pas-de-Calais), elle présente le double intérêt d'une région pionnière: elle offre un matériau empirique suffisant, tant en quantité (de nombreuses collectivités de la région ont mis en place une démarche de développement durable) qu'en qualité (le panel des collectivités ayant mis en place ce genre de démarche est suffisamment varié) ; elle permet de disposer d'un certain recul sur la mise en place des démarches.

Pour saisir la manière avec laquelle cette ressource cognitive latente se met en place à travers les stratégies publiques, nous avons sélectionné des institutions représentatives de l'intérêt commun et de la volonté publique : les collectivités territoriales (la région, les deux départements, des Etablissements Publics de Coopération Intercommunale, des communes), les services déconcentrés de l'État et des associations de collectivités (Groupements d'Intérêts Publics et pays). Au total, 21 institutions, dont les décisions apparaissent comme l'émanation légitime de la volonté publique, ont été investiguées entre septembre 2007 et mai 2008.

Nous avons essayé d'atteindre une certains représentativité territoriale, notamment en ce qui concerne le choix de la localisation des communes, des EPCI et des pays dans chacun des 
deux départements de la région. Les acteurs clés identifiés au sein de ces collectivités ont été interrogés au moyen d'entretiens libres, la méthode d'analyse textuelle aboutissant à restituer la structure des entretiens ${ }^{2}$. Les questions posées (sans guide précis, mais avec une technique de relance par mots-clés) concernaient la mise en application du développement durable (notamment les projets et/ou échecs emblématiques/remarquables), les difficultés rencontrées et la signification de la notion de développement durable... Pour disposer d'une vision la plus complète possible, les entretiens menés auprès des personnels techniques (21 au total) ont été complétés, lorsque cela a été possible, par ceux d'élus en charge de ces questions ( 5 au total). Dans deux communes, la personne en charge du développement durable était le directeur du cabinet du maire. Ces 28 entretiens ont ensuite été intégralement retranscrits en un corpus textuel analysé à l'aide de la méthode Alceste.

\subsection{La méthode Alceste}

La méthode de traitement Alceste permet d'aboutir à une classification des entretiens en «mondes lexicaux» (REINERT, 1993), c'est-à-dire en classes composées des termes considérés comme sémantiquement proches. L'originalité de la démarche repose sur cette conception de la proximité. Alors que la majorité des méthodes alternatives rapproche des termes ou des segments textuels en fonction de règles (par exemple grammaticales) ou selon un sens défini a priori (par exemple, au moyen de dictionnaires), Alceste part du postulat que le sens d'un mot vient du contexte dans lequel il est employé. Cela nécessite, dans l'analyse, de toujours replacer les mots identifiés par Alceste dans leur contexte, d'autant que si l'analyse peut être lemmatisée, il n'existe pas de procédure automatique de levée d'ambiguïté (pas de prise en compte de la polysémie). Cependant, comme le sens du texte provient de la proximité des mots entre eux, il n'y a pas d'intérêt à partir des mots eux-mêmes.

Sur un plan théorique, il y a un réel intérêt à utiliser la méthode Alceste en vue d'analyses en termes de proximité (PECQUEUR, et al., 2004, ToRRE, et al., 2008). La méthode de rapprochement et de distanciation des aspects sémantiques des discours apparait en effet comme une manifestation de la proximité cognitive encadrée par, et performant les repères territoriaux, géographiques et institutionnels. La méthode textuelle ne dispense donc pas d'analyses complémentaires (par exemple historiques) permettant de mieux éclairer la manière dont les contraintes institutionnelles, jouent par exemple contre la proximité cognitive. Elle nécessite un va et vient constant entre les résultats et le corpus analysé. 


\section{Figure 1 - Les quatre dimensions discursives des politiques locales de développement durable}

4258 uce classées

\begin{tabular}{|c|c|c|c|c|c|c|c|}
\hline \multicolumn{2}{|c|}{ Classe $1: 38,21 \%$} & \multicolumn{2}{|c|}{ Classe $3: 20,78 \%$} & \multicolumn{2}{|c|}{ Classe $2: 27,73 \%$} & \multicolumn{2}{|c|}{ Classe $4: 13,88 \%$} \\
\hline Formes & Khi2 & Formes & Khi2 & Formes & Khi2 & Formes & Khi2 \\
\hline choset & 140,2 & sociat1 & 356,1 & etat+ & 266,7 & communaut $<$ & 848,7 \\
\hline gens & 115,1 & economt & 340,7 & regiont & 251,8 & urbaint & 340,3 \\
\hline faire. & 114,0 & dura+ble & 252,1 & miset & 111,4 & communet & 294,7 \\
\hline venir. & 82,3 & energ +16 & 244,2 & agenda_21 & 110,1 & vice_president + & 257,5 \\
\hline truct & 67,7 & eaut & 175,3 & regionat1 & 108,1 & presidtent & 227,9 \\
\hline ça & 60,3 & ressourcet & 126,1 & nationa +1 & 99,9 & amenag+er & 225,3 \\
\hline diret & 59,0 & consommation & 122,5 & contrat + & 99,3 & delegationt & 137,8 \\
\hline fois & 50,2 & environnemental & 119,9 & cerdd & 95,4 & agglomeration< & 125,4 \\
\hline se & 50,0 & renouvel+er & 90,9 & acttion & 93,5 & charget & 103,1 \\
\hline ne & 45,5 & emploit & 86,4 & comitet & 91,6 & directeurt & 98,0 \\
\hline voir. & 44,0 & economt & 83,0 & strateg +16 & 85,1 & mairet & 77,1 \\
\hline reusstir & 42,7 & gestion< & 81,8 & pays & 74,3 & responsa< & 75,8 \\
\hline falloir. & 41,3 & liter & 74,8 & diren & 74,1 & bille & 68,7 \\
\hline aller. & 39,2 & developpem+ & 73,3 & projet+ & 73,9 & territoire+ & 67,0 \\
\hline mondet & 39,0 & urbanistme & 73,1 & outil +23 & 63,6 & compet+ent & 61,6 \\
\hline vous & 35,7 & question+ & 68,6 & differtent & 63,4 & arras & 60,0 \\
\hline prendre. & 33,9 & transportt & 66,0 & territoria +1 & 63,1 & directtion & 59,5 \\
\hline$q u+$ & 33,2 & besoint & 61,3 & directtion & 61,9 & elut & 58,7 \\
\hline moment+ & 31,8 & repondre. & 58,2 & operat< & 61,3 & missiont & 46,2 \\
\hline
\end{tabular}

Légende : Le signe $\alpha+»$ à la fin d'un mot signifie qu'il s'agit de sa forme lemmatisée. Lorsqu'un verbe est suivi d'un point, il regroupe l'ensemble des formes conjuguées. Le symbole $"<s$ indique que la forme présente lemmatisée, regroupe différentes catégories grammaticales (nom, adjectif...). La valeur du khi-deux n'a pas le sens habituel de mesure d'une erreur d'estimation ou d'application d'une loi de probabilité à un échantillon donné. Elle détermine la probabilité pour que l'appartenance d'un mot à une classe soit due au hasard (seuil de significativité : 3,84 ).

Les résultats de l'analyse ( $c f$. Figure 1) permettent de comprendre comment les décideurs publics locaux s'appuient sur la notion de développement durable dans la mobilisation collective. Cela passe par la réactivation de ressources cognitives latente, notamment issue de l'histoire régionale décrite dans la section 2. En mettant en perspective les difficultés pratiques de coordination de la décision publique sur le territoire (classe 3 ), objectif pourtant pointé du doigt dans la problématique du développement durable en particulier à l'échelle locale, les politiques locales insistent sur la nécessité de répondre de manière plus durable aux besoins des populations (classe 2). Ce faisant, elles font appel à l'histoire régionale et aux valeurs partagées qu'elle supporte dans un but de spécialisation et de différenciation, ce qui transparaît dans la mobilisation d'un vocabulaire rhétorique et exemplaire (classe 1). Cela ne va évidemment pas sans poser des problèmes de réorganisation et de changement institutionnel interne aux administrations locales (classe 4), très importants à certains égards, mais qui sont incidents à notre problématique. 


\section{- 5 - \\ Développement durable : une ressource cognitive légitime, active et différenciée}

L'interprétation de la classification permet de montrer comment la référence au développement durable est utilisée pour (re-)légitimer l'action publique dans un cadre territorialisé. Cela passe par la réactivation, via les politiques publiques, de ressources cognitives et en particuliers de valeurs en lien avec l'identité du territoire. Le développement durable devient alors une ressource pour le territoire qui, en reconfigurant son identité, contribue à spécialiser la région dans la maîtrise de cette ressource.

\subsection{Territorialisation de l'action publique et légitimité du développement durable}

La classe sémantique $\mathrm{n}^{\circ} 3$ permet ainsi de saisir la manière dont le développement apparaît comme une manière légitime de faire de la politique. Elle fait référence au territoire régional (local, urbain, rural, Nord Pas-de-Calais) ${ }^{3}$ comme unité spatiale cohérente de gouvernance publique en matière de développement durable. Le territoire est donné à voir, à travers ce vocable, comme un espace de coordination complexe (les échelles, les niveaux décisionnels) dans lesquels les différents niveaux de régulation sont enchevêtrés (GILLY, et al., 2005) et institutionnellement marqués par l'interdépendance des échelles de décision (FAVEREAU, et al., 1998). Les politiques de développement durable sont donc perçues comme une opportunité de redonner sens et cohérence au territoire, en dépassant les rigidités institutionnelles qui ont servi de cadre au développement industriel du siècle passé. Dans cette perspective, le morcellement administratif est perçu comme une difficulté qu'il faut contourner en inventant, ou en réinventant, des moyens de coopération (avec, ensemble, partager, coopérer). Plusieurs types de moyens sont mobilisés à cet effet : des moyens légaux (juridiques); des moyens institutionnels (pays, communaut+, intercomm+) ; des moyens relationnels (réseaux, partenaire) ; et enfin, un dernier moyen sans doute transversal à tous ceux identifiés, passe par la mise en place de projets.

Les collectivités s'interrogent ainsi sur le rapport des objectifs qu'elles souhaitent atteindre avec les moyens, notamment financiers (budget), dont elles disposent. Dans cette optique, la notion de développement durable est stratégiquement mobilisée de manière à inscrire l'action publique dans un cadre territorialisé intégré. Les savoirs sur lesquels il s'appuie sont de nature technique (les manières de faire), et ontique (les caractéristiques des acteurs et des outils des politiques publiques). Les premiers sont spécifiques au développement durable mais relativement peu stabilisés sur le plan institutionnel, alors que les seconds sont plus stables mais préexistent au développement durable. Néanmoins, elle s'apparente à une ressource: inscrire l'action publique dans le cadre du développement durable consiste, en redonnant une cohérence territoriale aux décisions, à améliorer leur efficacité tout en n'accroissant pas les dépenses publiques. Le développement durable, mobilisé dans cet argumentaire et configuré dans ces modalités d'actions territorialisées, apparaît ainsi comme une modalité légitime et acceptable de l'action publique. 


\subsection{L'activation politique d'une ressource latente}

En principe, la coïncidence de la volonté publique avec l'acceptabilité de politiques ne garantit pas l'efficacité de l'action publique. Il reste nécessaire que celles-ci soient effectivement acceptées (GADREY, 1994, OSTROM, 1996). La classe sémantique $n^{\circ} 2$ montre que l'acceptation repose sur la réactivation de certaines ressources cognitives, en particulier celles que nous avons qualifiées de déontologiques (les valeurs). Cela passe par la mobilisation d'un vocabulaire en référence aux différents secteurs des politiques locales, en particulier aux politiques sociales (logement, quartier, emploi...), de transports, d'urbanisme, etc., en lien direct avec les besoins des populations. L'emploi de termes propres aux thématiques environnementales (énergie, eau, ressources, déchets) est réinterprétée par les acteurs à l'aune du principe de transversalité ${ }^{4}$ (social, économique et environnemental). On y retrouve les termes par lesquels ce principe est institutionnellement appréhendé : volet, axe, thématique, dimension. Les questions d'évaluation des impacts à la fois environnementaux et socio-économiques des politiques mises en place confortent l'idée selon laquelle le principe d'intégration se trouve au cœur de cette classe sémantique. Celui-ci se décline finalement dans une vision de la politique publique en termes d'équilibre dont l'atteinte est identifiée comme une question, un problème difficile à traiter.

Cette dimension sémantique témoigne d'un certain pragmatisme à l'égard du développement durable. Les politiques locales s'appuient en effet sur des valeurs réactivées et sur une culture identitaire propre au Nord Pas-de-Calais. Ces politiques passent, notamment par une politique de valorisation du patrimoine minier et industriel. Trois axes politiques particulièrement édifiants peuvent être dégagés :

- Les politiques culturelles des collectivités de la région reposent sur la réhabilitation des infrastructures immobilières industrielles (LIEFOOGHE, 2010).

- Les politiques de logement s'appuient assez largement sur une réhabilitation de l'habitat minier (les corons).

- La politique de trame verte et bleue constitue un pan important de la politique environnementale de la Région, en s'appuyant sur l'ancien maillage territorial (via les liaisons ferroviaires entre les sites miniers)

Un principe commun guide l'ensemble de ces politiques. Il s'agit de réutiliser les infrastructures produites sous l'ère d'industrialisation, en valorisant ce patrimoine au regard du développement durable. La réutilisation des anciennes infrastructures industrielles à des fins culturelles, de logement, de préservation de l'environnement et de structuration du territoire se conçoit finalement dans une continuité historique. Le patrimoine minier ainsi que les valeurs dont il est porteur se trouvent ainsi remis en valeur et réactivé (COLLETIS, et al., 1993).

\subsection{Exemplification et rhétorique : affirmation du développement durable comme ressource régionale spécifique}

La mobilisation du développement durable et des ressources cognitives qui la soustende permet de mettre en évidence des modalités rhétoriques des politiques publiques. Ces modalités rhétoriques jouent sans doute un rôle dans un grand nombre de politiques publiques. Elles apparaissent toutefois assez manifestement lorsque la dynamique impulsée 
par la puissance publique implique de conséquents changements institutionnels, ce qui semble être le cas avec le développement durable. La classe sémantique $n^{\circ} 1$ met en évidence ces modalités rhétoriques. Le vocabulaire, assez pauvre sémantiquement, est tourné vers l'action, non seulement à travers la construction d'un système de croyances sur ce que font ou disent les gens, mais également avec la mise en œuvre des projets, jugés novateurs, de développement durable en particulier à travers les agendas 21 locaux (avancées, changer, faire). Il met en jeu trois types d'éléments rhétorique: la communication (dire, parler, demander), l'exemplarité (donner, prendre, servir d'exemple) et le prospectif (avancer, changer, réussir, agir). Ces trois éléments sont combinés dans un but persuasif et rhétorique (croire), non pas seulement pour inciter à bien agir, mais pour convaincre du bienfondé et de l'intérêt à adopter les valeurs et la démarche du développement durable. Dans cette optique, l'accent est particulièrement mis sur l'aspect concret des démarches et des projets de développement durable à destination d'autres acteurs. Il peut s'agir, soit d'instruire et d'éduquer le personnel technique des collectivités et/ou les citoyens à la démarche d'ensemble du développement durable (pédagog+), soit de partager des expériences. La mise en évidence d'exemple permet également de diffuser, non seulement des savoirs sur ce qui marche ou non en matière de développement durable, mais également des savoir-faire sur le mode du partage d'expériences.

La mise en évidence de cette dimension rhétorique du développement durable est fondamentale du point de vue de sa mobilisation en tant que nouvelle ressource identitaire. L'existence d'une base de savoirs partagés n'est pas suffisante pour constituer le développement durable en ressource identitaire, c'est-à-dire qu'elle ne se donne pas d'emblée comme étant «common knowledge », au sens de publicisation des savoirs (DUPUY, 1989). C'est cet aspect de la ressource cognitive qui se joue dans cette dimension sémantique. Ce faisant, la ressource activée dans les aspects sémantiques de politique publique (classe $n^{\circ} 3$ ) est non seulement révélée, mais également spécifiée dans sa dimension politique (COLLETISWAHL, 2002). Dans la classe $n^{\circ} 1$, l'image négative de la région est retournée pour en faire une ressource positive, mais également, spécialise la région (COLLETIS, et al., 2005). De fait, le savoir-faire des collectivités de la région en matière de développement durable constitue non seulement un trait caractéristique, mais également un domaine d'expertise reconnu à l'extérieur de la région.

\section{- 6 - \\ Convaincre, persuader et inciter : trois modalités complémentaires de l'action publique}

Les politiques locales de développement durable, en prenant appui sur le patrimoine issu de l'ère industrielle de la région, activent la durabilité comme ressource identitaire (re-) créant ainsi une base cognitive partagée. Cette manière de faire de la politique, qui s'appuie fondamentalement sur des modalités rhétoriques nécessite une forte adhésion des populations. Cette adhésion, ne relève pas seulement de l'assentiment ou de l'acceptabilité, mais également de la coproduction (GADREY, 1994, OSTROM, 1996). À cet égard, les politiques de développement durable se comprennent au regard de deux niveaux de prescription. Le premier entre dans le cadre habituel des incitations économiques et passe par un aménagement des procédures habituelles : introduction de clauses de durabilité dans les marchés publics, éco-conditionnalité des subventions, développement de filières durables par la demande publique, construction de logements HQE, etc. L'objectif de ces modalités prescriptives est de propager l'adoption de comportements durables en modifiant les habitudes de travail ou de 
fonctionnement des acteurs auxquels elles sont destinées, de créer les conditions d'une offre de produits durables sur le territoire en (re-)développant de nouveaux savoir-faire, d'aider à la consommation de produits durables, etc. Le développement territoriale durable repose alors, à ce niveau, sur les mécanismes institutionnels habituels des politiques économiques, sans mobiliser de nouveaux savoirs en matière de pratique politique ou de nouvelles ressources cognitives, au niveau des collectivités locales.

Au second niveau, complémentaire du premier, la durabilité sort du cadre marchand. L'incidence politique prend une valeur rhétorique (et non plus incitative). Il s'agit alors de considérer que le destinataire du service public a aussi un rôle à jouer dans sa production (GADREY, 1994, OSTROM, 1996). Autrement dit, toute politique publique et à plus forte raison de développement durable doit nécessairement, si elle veut être efficace, non seulement prendre appui sur la population, mais également la faire participer à sa gouvernance. De cette nécessité requise par l'efficacité de l'action publique, découle la nécessaire adhésion d'autres types d'acteurs (et en particulier des populations) à la coproduction du développement durable. En ce sens, les citoyens ne sont plus seulement considérés comme des consommateurs, mais aussi comme capables d'accepter ou non les arguments présentés pour les convaincre d'adopter une démarche soutenable.

\section{Figure 2 - Gouvernance politique du développement durable : un schéma d'apprentissage collectif normatif}

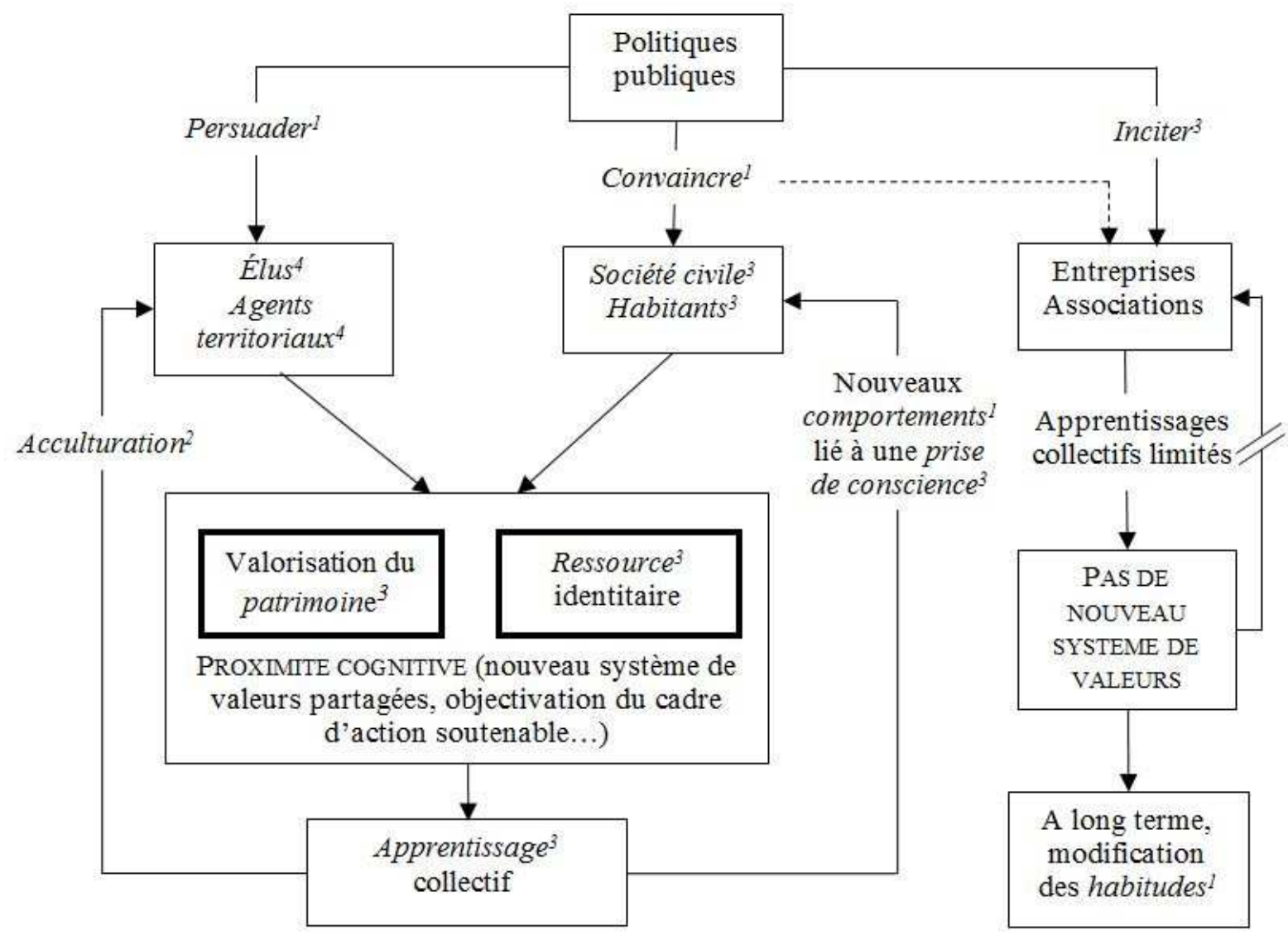

Légende : Les mots en italique ont été identifiés par Alceste. Le chiffre en exposant indique la classe sémantique à laquelle ils appartiennent. Le schéma détaille les leviers politiques d'apprentissage collectif en vue d'un développement régional durable. Des modalités d'action publiques (conviction et persuasion) sont mobilisées en complément des dispositifs incitatifs. Les premières prennent appui sur un patrimoine valorisé et sur une certaine identité territoriale (supports de proximité cognitive) et autorisent certains apprentissages collectifs. Dans le cas incitatif, ces apprentissages existent, mais ils restent relativement limités (lorsqu'ils sont impulsés par la puissance publique). 
La Figure 2 schématise la gouvernance politique du développement durable en mettant face à face les diverses modalités d'action publique sur lesquelles elle s'appuie, avec les différents types d'acteurs auxquels elle s'adresse. D'abord, l'incitation reste un mode d'interaction largement développé dans le cadre économique, en particulier avec le monde de l'entreprise, mais également avec d'autres collectivités, ou avec les associations. Ensuite l'aspect rhétorique s'adresse potentiellement à tous les acteurs. PERELMAN (1977) distingue ainsi l'auditoire universel formé de l'ensemble de ceux qui peuvent potentiellement entendre un discours, de l'auditoire particulier auquel il s'adresse directement. Cette distinction permet de mettre en évidence deux types de parties prenantes à la gouvernance politique du développement durable : l'auditoire particulier est constitué des agents territoriaux et des élus, premiers concernés par la bonne mise en application de la durabilité ; l'auditoire universel est formé des habitants, de la population, de la société civile, de l'ensemble des acteurs privés (y compris les entreprises), voire de l'ensemble des êtres humains. PERELMAN s'appuie sur une distinction conceptuelle pour mettre en évidence ce phénomène : les habitants doivent être «convaincus » du bienfondé de la démarche de développement durable ; les agents publics et élus doivent être «persuadés » qu'il s'agit d'une bonne politique, d'une bonne manière de faire ${ }^{5}$.

Chacune de ces modalités s'adresse donc de manière privilégiée (mais non exclusive) à une catégorie d'acteurs. L'incitation seule ne permet pas d'impulser un changement au niveau des valeurs collectives. Elle produit, au mieux, des effets d'apprentissages limités dans un «apprentissage en boucle simple» (ARGYRIS, 1993, p. 67): stratégies d'action conséquences - révision et adaptation du plan stratégique. Au mieux, on peut espérer une modification des comportements routiniers, dans un temps relativement long, en réaction à un signal incitatif. Ce schéma ne tient cependant pas compte du cas des entreprises investies dans des démarches responsables à l'égard de l'environnement, dont l'adoption d'une éthique environnementale ne répond principalement ni à une incitation (pas besoin d'incitation quand on est déjà convaincu), ni à des modalités rhétoriques (pas besoin d'être persuadé quand on a déjà changé nos comportements) (PLUMECOCQ, 2010).

En revanche, les modalités rhétoriques ont pour objectif de modifier en profondeur - au niveau des valeurs collectives, les raisons d'agir des acteurs. Le changement comportemental induit par ces modalités rhétoriques traduit donc un apprentissage collectif, facilité lorsque les « valeurs directrices » qui sous-tendent les stratégies d'action sont réinterrogées. C'est à partir de cette adhésion au nouveau système de valeurs, nécessitant une certaine force de conviction ainsi, comme nous l'avons montré qu'un certaine dose de savoirs partagés, que les individus seront capables de s'approprier et d'appliquer les enjeux et les pratiques du développement durable (ARGYRIS, 1993). Dans ce cas, l'adoption de valeurs durables se traduit, selon les termes significativement repérés par l'analyse textuelle : d'une part, au niveau des habitants et de la société civile, par l'adoption de nouvelles pratiques porteuses d'une proximité cognitive par lesquelles ils sont conduits à s'insérer de manière plus évidente dans la structure de pouvoir du territoire (TALBOT, 2008), notamment par l'adoption de démarches plus volontaires ; d'autre part, au niveau des agents territoriaux et des élus, par un changement de culture administrative (transversalité, participation, concertation, pensée globale, etc.). 


\section{-7 - \\ Conclusion}

Dans cet article, nous avons mis à jour les mécanismes politiques sur lesquels repose l'apprentissage collectif en vue d'un développement territorial plus durable : la valorisation d'un patrimoine et la (re-)définition d'une identité collective. Cela nous a permis de montrer que lorsque la puissance publique cherche à impulser un changement concernant l'ensemble du système territorial, elle mobilise des modalités rhétoriques, en complément des outils habituels de politique économique. Nous avons également montré que l'efficacité de ces modalités rhétoriques visant à modifier profondément les raisons d'agir des acteurs territoriaux prend appui sur un ensemble de ressources cognitives latentes qui lui préexistait. Au-delà du cas présenté ici, le papier entend donc insister sur la nécessité de replacer les problèmes de développement régional dans une perspective culturelle et identitaire, en particulier à travers une analyse historique.

Notre ambition était aussi, dans une démarche monographique, de mieux éclairer la mise en place de la gouvernance territoriale en Nord Pas-de-Calais, le rôle des pouvoirs publics locaux dans cette dynamique et l'appui sur la notion de développement durable. Dans la tradition comparatiste des études des trajectoires de développement, une voie de recherche importante nous semble ouvrir vers l'étude de ces moments de «jonction critique» d'institutionnalisation de choix contingents faits au regard d'une histoire passée et qui engagent vers une trajectoire de développement à venir (COLLIER, et al., 1991). Ainsi, pourquoi des régions qui ont connu, historiquement, un développement similaire à celui de la région Nord Pas-de-Calais (Lorraine, Ecosse...) n'ont-elles pas inscrit le développement durable au cœur de leur développement ? Et partant, sous quelles conditions le développement durable peut-il constituer une ressource identitaire pour le développement régional? L'hypothèse de déterminants non strictement économiques à ces jonctions critiques sur lesquels cet article a l'ambition d'insister, devra sans doute être privilégiée. Elle pourra ainsi interroger le caractère spécifique ou redéployable, au sens de COLLETIS, et al. (2005), de ce genre de ressource pour le territoire.

\section{Bibliographie}

ARgYris C (1993) Knowledge for Action. A Guide to Overcoming Barriers to Organizational Change. Jossey-Bass Publishers, San Francisco.

BARRÈRE C, BARTHÉlÉMY D, NIEDdU M, VIVIEN F-D (dir.) (2005) Réinventer le patrimoine. De la culture à l'économie, une nouvelle pensée du patrimoine. L'Harmattan, Paris.

BEAURAIN C (2008) La construction d'un territoire à partir des ressources environnementales : l'exemple de l'agglomération dunkerquoise. Géographie, économie, société, 10: 365-384.

Boltanski L, Thevenot L (1991) De la justification. Les économies de la grandeur. Gallimard, Paris.

Boschma R A (2005) Proximity and Innovation. A Critical Assessment. Regional Studies, 39: 61-74.

Boschma R A, Lambooy J G (1999) Evolutionary economics and economic geography. Journal of Evolutionary Economics, 9: 411-429. 
Bouba-Olga O, Grossetti M (2008) Socio-économie de proximité Revue d'Economie Régionale er Urbaine, 3: 311-328.

Brunet R, Ferras R, THERy H (1992) Les Mots de la Géographie. Dictionnaire critique. La Documentation française (cinquième édition, 2001), Paris.

COLLETIS-WAHL H-K (2002) Latence et spécification des facteurs de production. Quel rôle dans le développement régional ? Canadian Journal of Regional Science/Revue canadienne des sciences régionales, 25: 103-116.

Colletis G, Gianfaldoni P, Richez-BAtTesti N (2005) Territoire et économie sociale et solidaire : une mise en perspective. Recma, 296: 8-25.

Colletis G, PeCQueur B (1993) Intégration des espaces et quasi intégration des firmes : vers de nouvelles rencontres productives ? Revue d'Economie Régionale et Urbaine, 3: 489-508.

Colletis G, PeCQueur B (2005) Révélation de ressources spécifiques et coordination située. Economie et Institution, 6-7: 51-74.

COllier R B, COllier D (1991) Shaping the Political Arena: Critical Junctures, the Labor Movement, and Regime Dynamics in Latin America. Princeton University Press, Princeton.

DupuY J-P (1989) Convention et Common Knowledge. Revue Économique, 40: 361-400.

EMELIANOFF C (2005) Les agendas 21 locaux : quels apports sous quelles latitudes ? Développement durable et territoire. http://developpementdurable.revues.org/532

FAVEREAU O, QuiERS-VALETTE S (1998) Tous les problèmes d'incitation sont des problèmes d'interdépendance des niveaux de décision. In: VINOKUR A Décisions économiques, Economica, Paris, pp. 245-255.

GADREY J (1994) Les relations de services. In: DE BANDT J-D, GADREY J Relations de service, marchés de service, CNRS Éditions, Paris, pp. 23-42.

GADREY J, RUyters C, LAFFut M (2006) Des indicateurs régionaux de développement humain dans le Nord - Pas de Calais et en Wallonie. In: Conseil régional Etudes prospectives régionales, Direction de la Prospective, du Plan et de l'Évaluation, Lille.

GILLY J-P, WALLET F (2005) Enchevetrement des espaces de regulation et gouvernance territoriale : Les processus d'innovation institutionnelle dans la politique des pays en France. Revue d'Economie Régionale et Urbaine, 5: 699-722.

GREFFE X (2005) La culture et le développement local. OCDE, Paris.

KAHN R (2010) La dimension culturelle du développement territorial. Revue d'Economie Régionale et Urbaine, 4: 625-650.

Kant E (1781) Critique de la raison pure. Presses Universitaires de France, Paris (Édition, 1927).

LIEFOOGHE C (2010) Economie créative et développement des territoires : enjeux et perspectives de recherche. Innovations, 31: 181-197.

MAILLEFERT M (2009) Action collective territoriale et modèles de développement régionaux : le cas de trois sites de la Région Nord Pas-de-Calais. Vertigo, 9: http://vertigo.revues.org/8689

Noотевоом B (2000) Learning by Interaction: Absorptive Capacity, Cognitive Distance and Governance. Journal of Management and Governance, 4: 69-92. 
Ostrom E (1996) Crossing the Great Divide: Coproduction, Synergy, and Development. World Development, 24: 1073-1087.

Pascal B (1670) Pensées. Éditions Firmin Didot, Paris (Édition, 2004).

Pecqueur B, Zimmerman B (2004) Économie des proximités. Hermès Lavoisier, Paris.

PERELMAN C (1977) L'empire rhétorique. Rhétorique et argumentation. Librairie philosophique J. Vrin, Paris (Édition, 2002).

PlumeCOCQ G (2010) Organisations et développement durable : analyse comparée des discours d'acteurs locaux Économie et Institutions, 15: 31-57.

REINERT M (1993) Les "mondes lexicaux" et leur "logique" à travers l'analyse statistique d'un corpus de récits de cauchemars. Langage et Société, 64: 5-39.

Rist G (1996) Le développement. Histoire d'une croyance occidentale. Presse de la Fondation Nationale des Sciences Politiques, Paris.

SCARWELl H-J, KERGOMARD C, LAGANIER R (dir.) (2008) Environnement et gouvernance des territoires. Enjeux, expériences et perspectives en région Nord Pas-de-Calais. Presses Universitaires du Septentrion, Villeneuve d'Ascq.

TALBOT D (2008) Les institutions créatrices de proximités. Revue d'Economie Régionale et Urbaine, 3: 289-310.

TORRE A (2000) Économie de la proximité et activités agricoles et agro-alimentaires. Éléments pour un programme de recherche. Revue d'Économie Régionale et Urbaine, 4: 407426.

TORRE A (2008) Commentaire : Réflexions à partir des textes de O. Bouba-Olga, M. Grossetti et D. Talbot. Proximité géographique et pragmatique de l'action. Revue d'Economie Régionale et Urbaine, 3: 329-332.

Torre A, Rallet A (2005) Proximity and localization. Regional Studies, 39: 47-60.

TORRE A, ZuindeAU B (2008) Économie de la proximité et environnement : état des lieux et perspectives. Canadian Journal of Regional Science/Revue canadienne des sciences régionales, 31: 133-160.

VIVIEN F-D (2009) Pour une économie patrimoniale des ressources naturelles et de l'environnement. Monde en développement, 145: 17-28.

Zuindeau B, Goxe A, Villalba B (2008) Séquelles environnementales et persistance du territoire. In: SCARWELl H-J, KERGOMARD C, LAGANIER R, Environnement et gouvernance des territoires. Enjeux, expériences et perspectives en région Nord Pas-de-Calais, Presses Universitaires du Septentrion, Villeneuve d'Ascq, pp. 89-107.

\section{Notes}

1 - Quatre communes sont particulièrement en avance en matière de développement durable : Lille (adhésion à la Charte d'Aalborg dès 1995) ; Dunkerque (principe d'intégration pris en compte dans le Projet d'Agglomération de 1996); Loos-en-Gohelle (le développement durable est au cœur de la Charte de la Qualité de Vie signée en 1996) ; et Valenciennes (lancement de l'agenda 21 local en 1998).

2 - Le recours à la méthode Alceste nécessite donc certaines précautions d'usages. Il reste nécessaire de définir dès la phase pré-analytique durant laquelle les techniques d'entretiens sont envisagées, les objectifs poursuivis en utilisant Alceste. 
3 - Dorénavant les mots en italique feront référence aux formes analysées et restituées par le logiciel.

4 - Le principe d'intégration consiste à prendre simultanément en considération les conséquences sociales économiques et environnementales d'une action entreprise.

5 - La différence entre la conviction et la persuasion fait l'objet de vifs débats. Plusieurs traditions s'opposent en philosophie. Pour PASCAL (1670), par exemple, la conviction tient de la raison, tandis que la persuasion tient de la passion, tandis que pour KANT (1781), la première est objective, tandis que la seconde est subjective. 